\title{
Automated contact angle estimation for three-dimensional X-ray microtomography data
}

\author{
Katherine A. Klise ${ }^{\text {a }}$, Dylan Moriarty ${ }^{\text {a }}$, Hongkyu Yoon ${ }^{\text {a }}$, Zuleima Karpyn ${ }^{\text {b }}$ \\ ${ }^{a}$ Geoscience Research and Applications Group, Sandia National Laboratories, Albuquerque, NM 87185 \\ b John and Willie Leone Family Department of Energy and Mineral Engineering and EMS Energy Institute, \\ The Pennsylvania State University, University Park, PA 16802
}

\begin{abstract}
Multiphase flow in capillary regimes is a fundamental process in a number of geoscience applications. The ability to accurately define wetting characteristics of porous media can have a large impact on numerical models. In this paper, a newly developed automated three-dimensional contact angle algorithm is described and applied to high-resolution X-ray microtomography data from multiphase bead pack experiments with varying wettability characteristics. The algorithm calculates the contact angle by finding the angle between planes fit to each solid/fluid and fluid/fluid interface in the region surrounding each solid/fluid/fluid contact point. Results show that the algorithm is able to reliably compute contact angles using the experimental data. The in situ contact angles are typically larger than flat surface laboratory measurements using the same material. Wetting characteristics in mixed-wet systems also change significantly after displacement cycles.
\end{abstract}

\section{Keywords}

Contact angle, Multiphase, Wettability, X-ray microtomography

\section{Introduction}

Prediction of multiphase flow in geologic materials is crucial to understanding, developing and exploiting subsurface systems including emerging geological activities such as enhanced oil recovery [1, 2] and geologic $\mathrm{CO}_{2}$ storage [3]. Pore-scale understanding is crucial for quantifying multiphase flow considering complex pore geometry, changes in phase saturations, chemical reactions at different phase interfaces, and trapping of residual phases [4-6]. Multiphase flow behaviors in porous media are often characterized using capillary pressure, fluid saturation, and relative permeability [7]. Capillary pressure is a function of pore geometry and wetting properties and is defined as the pressure difference across the interface of two different fluids. Wettability is defined as a fluid's tendency to adhere to a solid surface in the presence of another fluid [8] and can be quantified by the contact angle formed at the interface between two immiscible fluids and the solid surface. Typically, contact angles are measured on a flat surface of solid material using a variety of techniques [9]. The contact angles measured on a flat surface often fail to capture the arrangement of fluids at pore scale due to heterogeneous characteristics such as mineral composition, geometry, and surface roughness [5, 10-12]. Additionally, contact angle values change under drainage and imbibition cycles (i.e., contact angle hysteresis) [13-15] and due to chemical reactions [11]. The ability to accurately define wetting characteristics of porous media can have a large impact on predicting multiphase behavior in porous media.

Recent advances in high-resolution imaging techniques such as X-ray microtomography (micro-CT) provide an opportunity to characterize in situ fluid distributions in pore structures under varying pressure, temperature, salinity, and/or wetting conditions [5, 16-19]. With micro-CT data obtained in a water-wetting bead packing column, Armstrong et al. [20] demonstrated a curvature-based method to analyze contact angles and linked the capillary pressures calculated from the curvature measurements to those from transducer-based measurements. Their work also shows the importance of connected fluid on curvature measurements. Andrew et al. [21] measured pore-scale contact angles for a supercritical $\mathrm{CO}_{2}$-brine-carbonate system at reservoir conditions using micro-CT data, showing that the 
range of contact angles measured for manually selected image sets can be attributed to contact angle hysteresis and surface heterogeneity. Brown et al. [22] use mirco-CT data to demonstrate the importance of segmentation methods when measuring interfacial characteristics. Additionally, they are able to track wetability alteration over the course of mutli-phase flow experiments. These recent studies demonstrate the value of three-dimensional (3D) image datasets to investigate pore-scale contact angle hysteresis under varying conditions.

The objectives of this work are to present a new 3D contact angle algorithm to automatically measure in situ contact angles and apply the algorithm to published results of multiphase flow experiments imaged by micro-CT under different wettability conditions. The algorithm avoids manual calculations from selectively chosen image sets or interpolated surface curvature. This work utilizes experimental data from Celauro et al. [18], which includes fluid distributions in uniform and mixed grain core samples of soda lime glass and polyethylene plastic beads. To account for the impact of hysteresis, contact angles are calculated after advancing and receding displacement cycles. Results are related to the wetting properties during each displacement cycle and compared to contact angles measured on a flat surface using the same solid materials. The accuracy of the new algorithm is demonstrated using a synthetic test case and implications of the 3D contact angle analysis are discussed.

\section{Materials}

Experimental data used in this paper are taken from a study by Celauro et al. [18] which looked at the pore scale effects of variable wettability on multiphase fluid flow. The experiments used core samples that measured approximately $25 \mathrm{~mm}$ in diameter and up to $102 \mathrm{~mm}$ in length. Each core was packed with beads of varying wettability but uniform grain diameter ranging from 0.710 to $0.850 \mathrm{~mm}$. A micro-CT scanner was used to image solid and fluid distribution during a series of displacement cycles. The voxel resolution for these experiments ranged from 0.0266 to $0.0287 \mathrm{~mm}$ in both the horizontal directions. Digital core slices ranged from 0.0268 to $0.0278 \mathrm{~mm}$ in thickness. The 3D data is then constructed by stacking the slices in the vertical direction. This results in approximately 2.5 billion data points for each experiment. For this research, contact angles are calculated within a volume of interest measuring $13.7 \mathrm{~mm} \times 13.7 \mathrm{~mm} \times 27.4 \mathrm{~mm}$ ( $500 \times 500 \times 1000$ voxels $=250$ million voxels) in the center of each bead back. A central region of the bead pack was selected to avoid edge effects. The experiments use brine and kerosene as the wetting or non-wetting phase depending on the bead type used in the experiment. Fluid properties correspond to those reported by Landry et al. [23].

The bead packs used in this study consist of varying amounts of polyethylene plastic beads (oilwetting) and soda lime glass beads (water wetting). The first bead pack was made up entirely of plastic beads. A flat plate of the same plastic material was used to measure the contact angle using the sessile drop method. Results showed the plastic material was intermediately wetting with a measured contact angle of $100.9^{\circ}$. The second bead pack was made up entirely of glass beads. The dynamic sessile drop technique was used to determine the contact angle on a flat plate of glass. The contact angle varied from $77.1^{\circ}$ to $44.8^{\circ}$ in the space of 2 minutes and had a median contact angle of $47.7^{\circ}$. The third bead pack was filled with a mix of plastic and glass beads. This sample consisted of $57.1 \%$ glass and $42.9 \%$ plastic beads by volume. While the beads were uniformly mixed before packing the core, the plastic beads tended to group together along the outer edge of the core sample due to static charge. Considering the percent of each type of bead and the contact angle measurements on flat surfaces, the bead pack is expected to be slightly water-wet on average.

Each bead packed core was set in the micro-CT scanner. The scanner was used to map the interior structure of the bead pack, as well as the residual fluid distribution in the pore space. After placement in the scanner, an initial dry scan was taken to observe the grain distribution within the core. Next, each core was saturated with either brine or kerosene. The glass bead pack and the plastic bead pack were saturated with their respective wetting fluid (brine and kerosene, respectively). The mixed glass/plastic 

brine was displaced by kerosene (or vice versa in the case of the plastic bead pack). After the system reached steady state, a micro-CT scan was taken to determine the distribution of brine and kerosene in the core. Finally, the system underwent a $2^{\text {nd }}$ displacement where kerosene was displaced by brine (or vice versa in the case of the plastic bead pack). When the system reached steady state, a final scan was taken to measure the distribution of brine and kerosene. Contact angle calculations in this study come from micro-CT scans after both the $1^{\text {st }}$ and $2^{\text {nd }}$ displacements. For the uniform glass and plastic bead packs, the displacement cycles are setup to replicate the standard process of drainage for the $1^{\text {st }}$ displacement and imbibition for the $2^{\text {nd }}$ displacement.

Figure 1 illustrates residual saturation and vertical cross-section for the central volume of interest for each of the three bead packs. The direction and fluid type for displacement is shown for each crosssection. For each experiment, the $1^{\text {st }}$ and $2^{\text {nd }}$ displacements result in fairly even residual saturation profiles. In the uniform systems, it is expected that the residual wetting phase will be trapped in small pores after drainage and the residual non-wetting phase will be trapped in large pores after imbibition. With the uniform glass and plastic bead packs, the cross-sectional view after drainage and imbibition show this characteristic. With the mixed glass/plastic bead pack, the cross-sectional view after $1^{\text {st }}$ displacement shows that the residual fluid (brine) is often in contact with glass beads and fills small pore spaces. After the $2^{\text {nd }}$ displacement, the residual fluid (kerosene) is in contact with glass and plastic beads and fills a range of pore configurations. The ability to measure contact angles within 3D experimental data could help explain this phenomenon.

Micro-CT data records a CT registration value at each voxel. The CT registration value depends on the radiodensity of the material which depends largely on density [24]. To use this type of data to measure contact angles, each voxel in the data set must be categorized as one of four possible phases: glass, plastic, brine, or kerosene (as shown in Figure 1). To segment the data into distinct phases, threshold values were determined using frequency distributions for each experiment [18]. The initial scan, taken when the core was dry, was first segmented into glass, plastic, and pore space using simple thresholding. Once the glass and plastic phases were defined, the brine and kerosene phases were superimposed on the segmented pore structure according to the fluid distributions revealed by CT scans after $1^{\text {st }}$ and $2^{\text {nd }}$ displacements. The results are volumetric datasets of each bead pack, at the end of each displacement, showing the spatial location of plastic, glass, kerosene, and brine voxels. In cases of ambiguous phase identification (i.e. the same voxel was defined as glass and brine), voxels are left as undefined. Such undefined voxels only occurred in the mixed glass/plastic bead pack and accounted for less than $0.70 \%$ of the analyzed data. For this reason, very few undefined voxels are visible in Figure 1. The experimental data will be made available upon request. 
2nd Displacement (Imbibition)

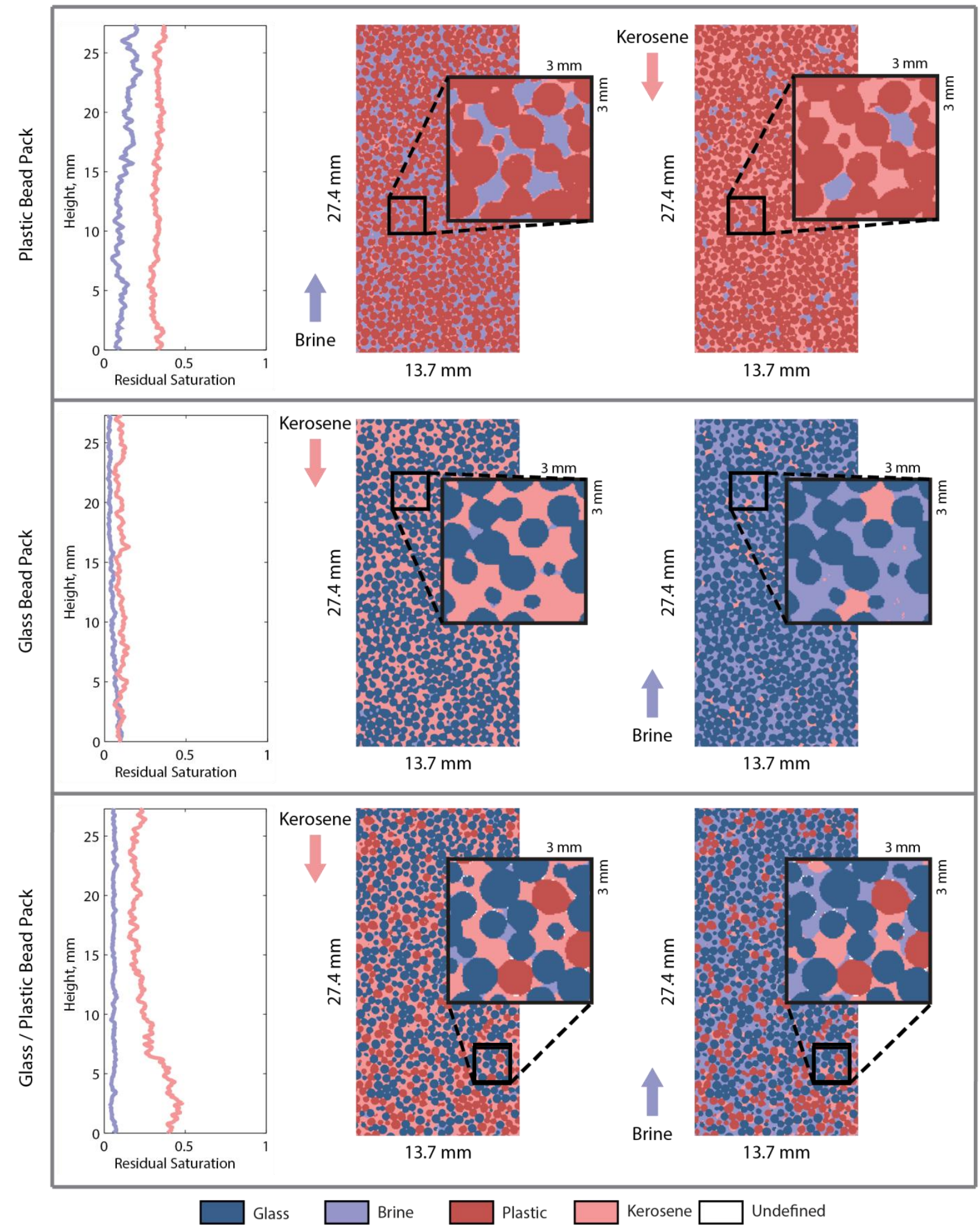

Figure 1 - Residual saturations and vertical cross-sections for each bead pack. The displacement direction is represented by arrows to the left of each cross-section. Modified from Celauro et al. (2014) 


\section{Methods}

In this section, the algorithm for the 3D contact angle calculation is described and its use with the experimental data is explained. Additionally, a synthetic test case is used to validate the algorithm.

\subsection{D contact angle algorithm}

The 3D contact angle algorithm used in this work is a new method developed to directly use 3D segmented data without post-processing. Due to the large volume of image datasets, which typically contain over 100 million voxels in a core sample, the automation of the algorithm is key to avoid manual calculations from selectively chosen image sets. The 3D data is typically obtained from segmented micro-CT images as in this work, but not limited to any particular system. To calculate contact angles, a solid phase and a light and dense liquid phase must be defined. Using this algorithm, the angle is calculated through the denser fluid phase. In the algorithm description below, the dense fluid phase is brine and the light fluid phase is kerosene, in keeping with the experimental data described in Section 2. The experimental data includes two solid phases: glass and plastic. The algorithm can track multiple solid phases to associate contact angle measurements with specific solids, as described below. The entire algorithm was developed using Matlab with the image processing toolbox (Matlab v8.2.0.701, R2013b).

In this algorithm, the first step is to define the interface between kerosene and brine and between solid (i.e., glass or plastic) and brine, along with contact points where the solid, brine, and kerosene intersect. Interfaces are equivalent to phase boundaries in a 3-phase diagram while contact points are equivalent to triple points in a 3-phase diagram. To illustrate this, a $15 \times 15$ voxel cross-section from the 3D experimental data is shown in Figure 2. Interfaces are identified by isolating brine voxels that share a face with a solid voxel or a kerosene voxel. Other types of connectivity, including edge or corner connected, could also be used in the algorithm. However, restricting the interface to voxels that share a face ensures that the algorithm only uses voxels in direct contact with the brine phase. If a brine voxel shares a face with a solid voxel, that brine voxel is assigned to the solid/brine interface. Similarly, the kerosene/brine interface is formed by brine voxels that share a face with kerosene. Contact points, the location where contact angles can be calculated, are defined as brine voxels that share a face with both the solid and kerosene phase. Using this grid-based method, all contact points and interface points are assigned to the voxels in the outer boundary of the brine phase. While other contact point and interface assignments are possible (for example, the brine/solid interface could be assigned to the solid), assigning all contact and interface points to the brine phase provides a consistent way to preserve the geometry surrounding each contact point. 

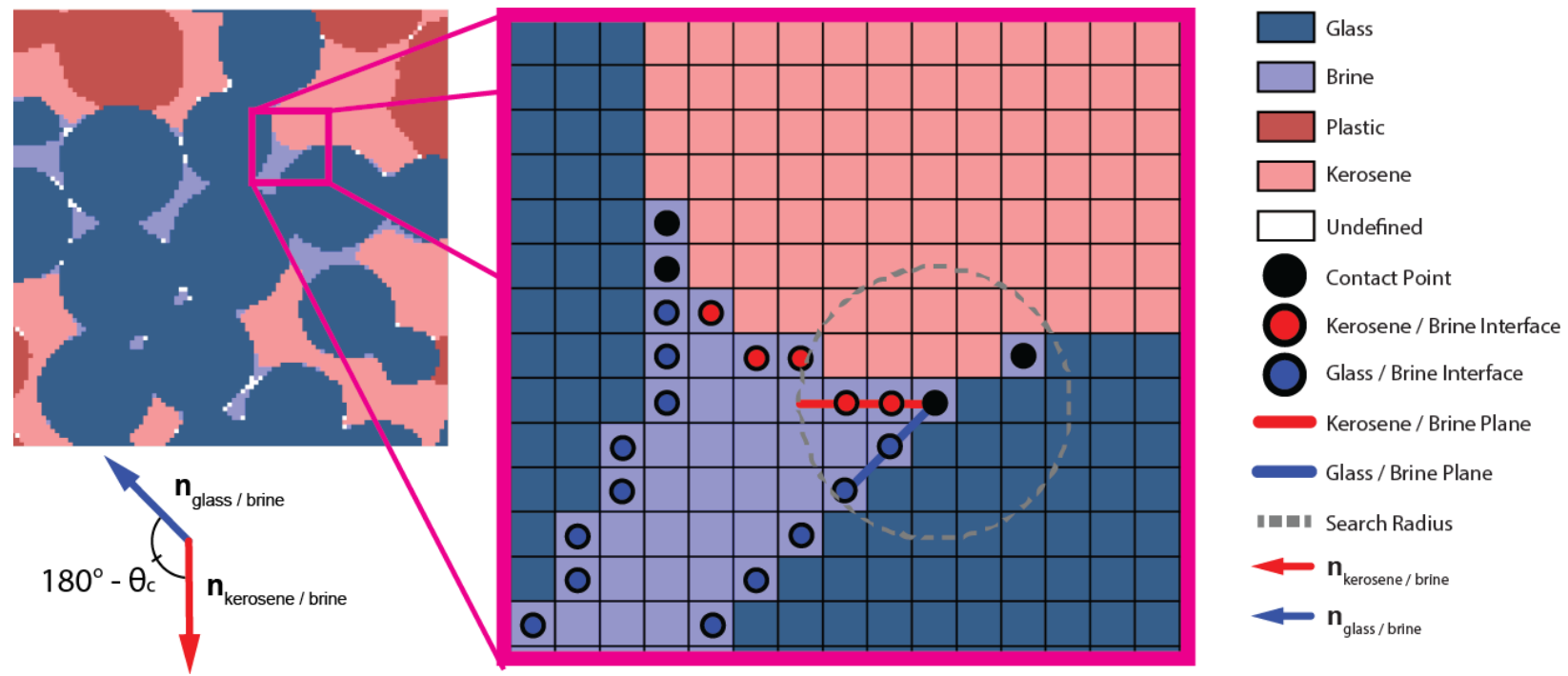

Figure 2 - Two-dimensional view of interface identification, contact points and contact angle calculation

Any contact points near the edge of the sample are discarded to prevent boundary effects during the contact angle calculation. For data sets with multiple solid phases, like the experimental data with glass and plastic beads, the contact points are divided into points associated with each solid phase. The solid/brine interface is also divided into interfaces for each solid phase (i.e. glass/brine interface and plastic/brine interface). This allows contact angles to be associated with specific solids.

Once all interface and contact points are located, the contact angle is calculated at each contact point. A plane is fit to each interface (kerosene/brine and solid/brine) within a search volume surrounding a contact point. This volume should be kept small to ensure that the contact angle reflects the tangent angle at the contact point. The interface points within this search volume must form a connected surface with the contact point (defined as a surface that shares at least one corner with its neighbors) to be used in the calculation. This discounts voxels along the interface that are isolated from the contact point or may be part of the interface with adjacent solids or fluids. At a minimum, 3 points must be available to fit a plane to the interface. To ensure that the interface is adequately represented by the available data, more than 3 points can be required. The search radius and minimum number of points can also be changed for different systems.

Finally, the contact angle is computed by taking the dot product of the normal vectors to each plane. For correct calculation, the direction of both normal vectors must meet certain criteria. If both vectors point in the direction of the brine phase, then the contact angle is the supplement to the angle created by the two vectors. Any vector that does not point in the direction of the brine is multiplied by -1 to make the normal vector equal and opposite. If an appropriate direction for each normal vector cannot be established, then the contact point is considered to be invalid. This occurs when the brine phase is one voxel thick in the region surrounding the contact point. For valid contact points, the contact angle $\left(\theta_{c}\right)$ is then computed as:

$$
\theta_{c}=180^{\circ}-\operatorname{acos}\left(\mathbf{n}_{\mathrm{solid} / \mathrm{brine}} \cdot \mathbf{n}_{\mathrm{kerosene} / \mathrm{brine}}\right)
$$

where $\mathbf{n}_{\text {solid/brine }}$ is the normal vector to the plane fit to the solid/brine interface, and $\mathbf{n}_{\text {kerosense/brine }}$ is the normal vector to the plane fit to the kerosene/brine interface. The process for calculating contact angles is repeated for each contact point over the entire dataset. 
Using the micro-CT data described in Section 2, contact angles are calculated after both the $1^{\text {st }}$ and $2^{\text {nd }}$ displacement cycles. In Figure 2, the isolated contact point would be considered invalid unless the point is connected to the glass/brine and kerosene/brine interface in the third dimension. The figure also shows two contact points next to each other. In that configuration, both contact angles are calculated using their respective search radii and slight variations are expected. For this example, all contact points are assigned to the glass phase.

\subsection{Validation and sensitivity analysis of the 3D contact angle algorithm}

To test the accuracy of the newly developed method, the algorithm is applied to a synthetic test case where the contact angle is analytically defined. The test case represents a single spherical solid in contact with brine along a diagonal plane (Figure 3). In the figure, kerosene is represented by the white (void) space. Contact points form a rim around the sphere where the solid, kerosene, and brine intersect. Based on the location of the kerosene/brine interface with respect to the sphere, contact angles can be analytically defined by assuming a perfect sphere and smooth interface. In the test case, the kerosene/brine interface is shifted from the bottom left corner to the upper right corner to cover the full range of contact angles. Using this configuration, the theoretical contact angle decreases from $180^{\circ}$ to $0^{\circ}$. The theoretical contact angle is $90^{\circ}$ when the brine phase covers half of the sphere. As the location of the kerosene/brine interface shifts, the algorithm is applied to calculate the contact angle along the corresponding rim.
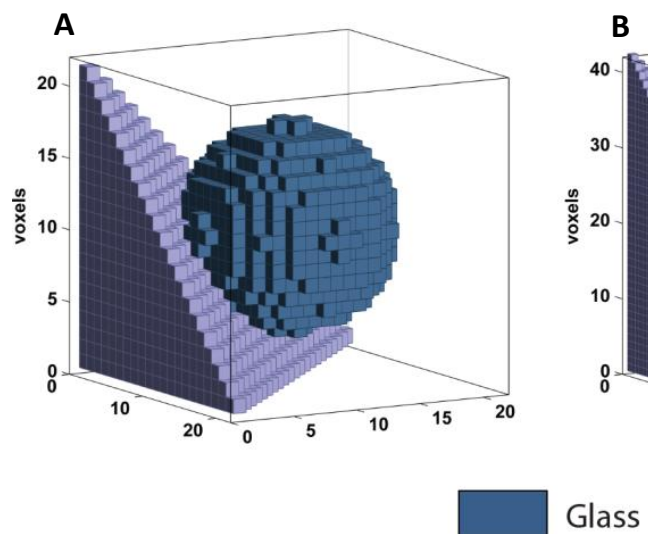

B

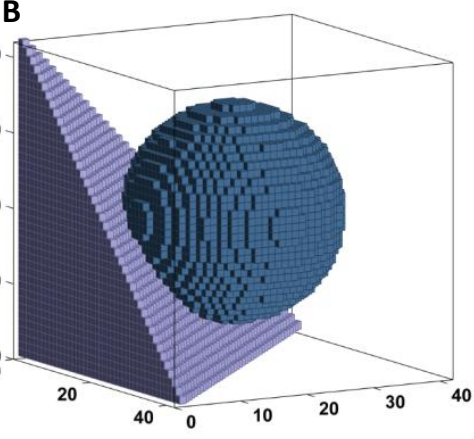

Brine

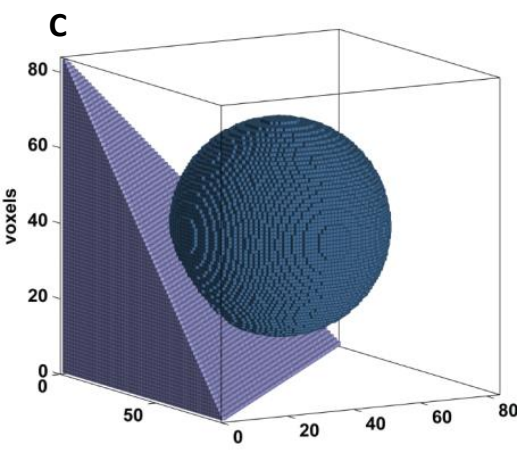

Kerosene

Figure 3 - Example test cases with a spherical bead at three different voxel resolutions: A) $1 / 14^{\text {th }}$, B) $1 / 28^{\text {th }}$ and C) $1 / 56^{\text {th }}$ of the sphere diameter. The diagonal plane represents the brine phase, at a theoretical contact angle of approximately $160^{\circ}$.

Using this test case, the sensitivity of 5 parameters are evaluated, these parameters include: data resolution, search radius, minimum number of points to fit a plane to the interface, types of connectivity, and noise at the interface. Three resolutions are defined by varying the number of voxels across the sphere (as shown in Figure 3 ). The length scale of 1 voxel is set equal to $1 / 14^{\text {th }}, 1 / 28^{\text {th }}$ and $1 / 56^{\text {th }}$ of the sphere diameter (a voxel resolution equal to $1 / 28^{\text {th }}$ of the sphere diameter approximates experimental conditions). The search radius is set to be approximately $5 \%, 10 \%$, and $15 \%$ of the sphere diameter for each resolution (rounded up to the nearest voxel). The minimum number of points required to fit a plane to the interface is set to 3,5 , and 7 . The analysis includes 3 types of connectivity: 6-connected voxels are neighbors to every voxel that touches a face, 18-connected voxels are neighbors to every voxel that touches a face or edge, and 26-connected voxels are neighbors to every voxel that touches a face, edge, or corner [25]. Connectivity is used to define the contact points and interfaces. 
Noise is added to the solid/fluid and fluid/fluid interface as defined by a percentage of the surface area, $0 \%, 10 \%$, and $20 \%$ noise are used. Noise is added to track the impact of imperfect segmentation. While segmentation error will not be randomly distributed, adding random noise tracks how changes to the interface impact the contact angle calculation. Future research will focus on the impact of specific segmentation methods on the contact angle measurements. Overall, the sensitivity analysis includes 243 combinations. Five realizations are included to capture the impact of noise.

For each combination, the theoretical contact angle is compared to the calculated contact angles by calculating the root mean square error (RMSE) for contact angles between 0 and $180^{\circ}$. Figure 4 shows the impact of changing resolution, search radius, minimum number of points, connectivity, and noise at the interface. The mean RMSE is plotted on the $y$-axis. As expected, increasing resolution increases the accuracy of the calculated contact angles, given that both the sphere and interface are pixelated representations of a perfect sphere and smooth interface. The accuracy of the calculated contact angles also improves as search radius increases. However, at low resolution, the improvement is minimal. Given that most interfaces have more than the minimum number of points available for the contact angle calculation, the minimum number of points has a small impact on the results. As expected, adding noise to the interface has a negative impact on the accuracy of the contact angle calculation. At the resolution of experimental data, $10 \%$ misclassified voxels at the interface can decreases the accuracy of the contact angle calculation by $8^{\circ}$. With $20 \%$ misclassified voxels, accuracy can decrease by $12^{\circ}$. As compared to 18 and 26-connected voxel connectivity, using 6-connected voxel connectivity results in the best estimate of contact angles. 6-conntected, or face, connectivity ensures that voxels are in contact over the largest surface area.

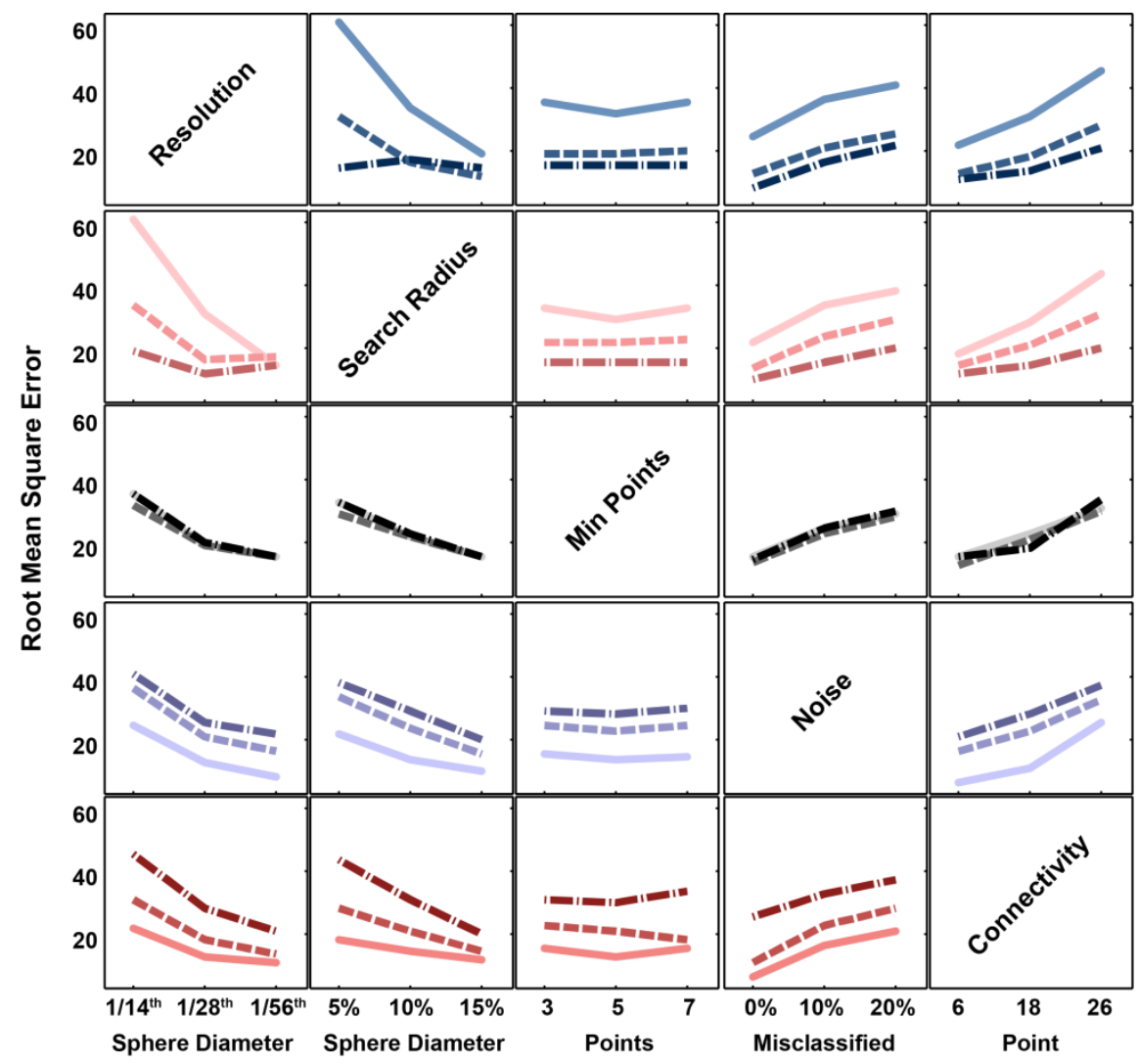

Resolution

$1 / 14^{\text {th }}$ Sphere Diameter -ோ=ே+ $1 / 28^{\text {th }}$ Sphere Diameter "-1/56

Search Radius

$5 \%$ Sphere Diameter

- $10 \%$ Sphere Diameter ロー・・ 15\% Sphere Diameter

Min Points

3 Points

-r=mer 5 Points

ロ・ー 7 Points

Noise

$0 \%$ Misclassified

=ோ=ー1 $10 \%$ Misclassified

"ー・- 20\% Misclassified

Connectivity

6 Point

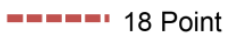

ロ・・ 26 Point

Figure 4 - Sensitivity of resolution, search radius, minimum number of points to fit a plane to the interface, added noise at the interface, and connectivity using the spherical bead test case. 
Figure 5 compares theoretical and calculated contact angle measurements for the three resolutions. The search radius is set to $10 \%$ of the sphere diameter, the minimum number of points is set to 5 , face connectivity is used, and no noise is added at the interfaces. Based on the resolution and search radius, there are a discrete number of plane orientations that can be defined using this grid-based approach. Despite this limitation, the test case demonstrates that the algorithm is able to replicate a wide range of contact angles over the range of resolutions tested here. Due to the stair-wise voxel configuration (see Figure 3) the calculated contact angle values around the contact rim are not a single value, but a distribution. The calculated contact angle (the median value along with the $90 \%$ confidence interval) is plotted for each theoretical angle. The plots include contact angles that can be measured using all three resolutions. Comparison demonstrates that the newly developed algorithm replicates a wide range of contact angles with high accuracy. For all three resolutions tested, the algorithm tends to overestimate contact angles. This is attributed to the grid based representation of the interfaces. The effect is greatly diminished as the resolution increases to $1 / 56^{\text {th }}$ of the sphere diameter. At the higher resolution, the median contact angle closely approximates the theoretical angle and the confidence interval is very small. It is important to note that the perimeter of the contact rim decreases as the contact angle approaches $0^{\circ}$ and $180^{\circ}$, which reduces the number of points used to calculate the median and confidence interval. For the $1 / 14^{\text {th }}, 1 / 28^{\text {th }}$ and $1 / 56^{\text {th }}$ voxel resolution, the RMSE between the theoretical and calculated contact angles are $13.2^{\circ}, 7.3^{\circ}$, and $5.7^{\circ}$ with $90 \%$ confidence intervals of $13.3^{\circ}, 9.3^{\circ}$, and $2.7^{\circ}$, respectively. The RMSE for these cases are lower than the values in Figure 4 because all fitting parameters are held constant.

These results demonstrate that even a simple test case includes complex geometries that makes contact angle calculation challenging. The 3D contact angle algorithm is able to calculate contact angles in a wide range of conditions. Based on this test, the contact angle algorithm is expected to give reliable results using the experimental resolution.
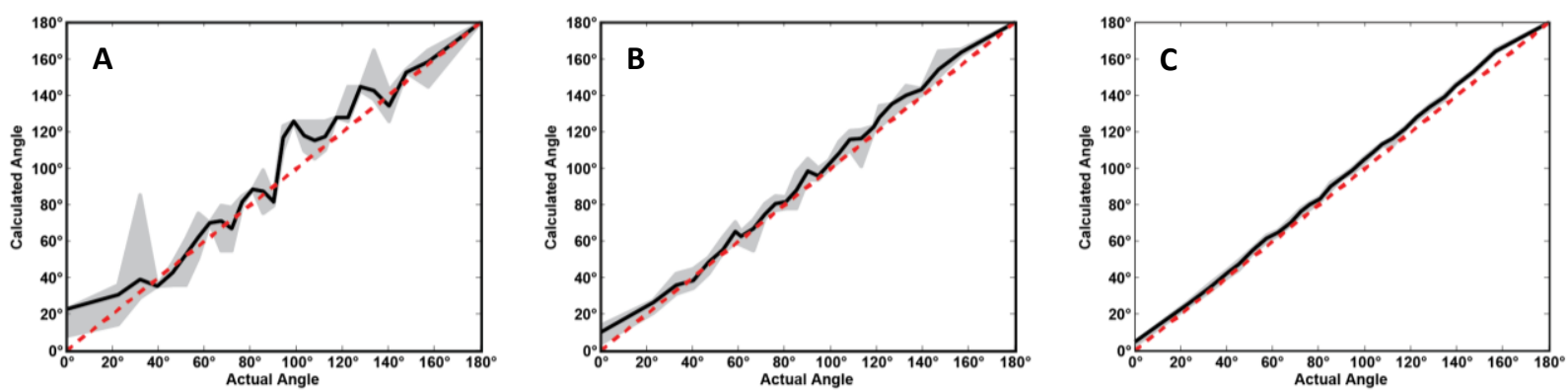

Figure 4 - Theoretical contact angle compared to the calculated contact angle at three different voxel resolutions: A) $1 / 14^{\text {th }}$, B) $1 / 28^{\text {th }}$ and C) $1 / 56^{\text {th }}$ of the sphere diameter (as shown in Figure 3 ). Search radius is set equal to $10 \%$ the bead diameter, the minimum number of points is set equal to 5 , face connectivity is used and no noise is added. The median is represented as a black line, while the $90 \%$ confidence interval is represented by the gray region. The red dashed line in dicates a 1:1 correlation.

\section{Results and Discussion}

The contact angle algorithm described in this paper was applied to the segmented data of pore-scale distribution of brine and kerosene in glass, plastic and mixed bead packs from Celauro et al. [18]. Several thousand contact angles are calculated within the volume of interest selected from each experiment. The distributions of calculated contact angles are presented in Figure 6. Figure 6a includes contact angles calculated using the glass and plastic bead packs with uniform-wetting properties. Figure 
$6 \mathrm{~b}$ combines the glass and plastic contact angles calculated from the mixed glass/plastic system. In both cases, there are four distributions that distinguish contact angles associated with glass and plastic after the $1^{\text {st }}$ and $2^{\text {nd }}$ displacement. Table 1 and Table 2 include information on the number of contact points, number of invalid points, and statistics on the contact angle distribution for each bead pack. Contact points are classified as invalid if the connected interface does not contain enough points within the search radius, or if the normal vector direction cannot be established. Some bead packs have a large percentage of contact points classified as invalid. Future research will focus on how to reduce the number of invalid points, which are possibly caused by misclassified voxels in the segmented data in combination with the resolution of the experimental data. With the exception of contact angles on plastic beads in the mixed glass/plastic bead pack after the $1^{\text {st }}$ displacement (Table 2 , column 1 ), there are still over 142,000 points used to calculate the contact angle distribution in each case.
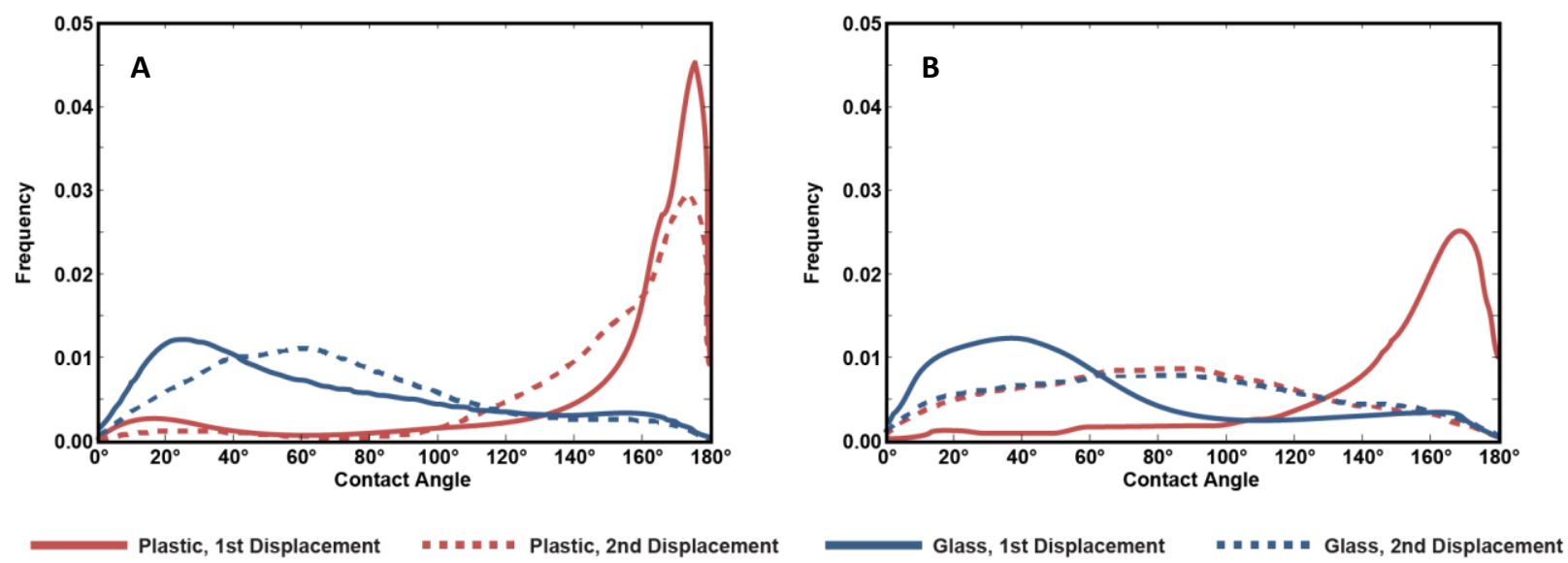

Figure 5 - Probability distribution functions for contact angles in A) uniform-wetting experiments (glass bead pack and plastic bead pack) and B) mixed-wetting experiment (glass/plastic bead pack)

Table 1 - Contact angle statistics for the uniform-wetting experiments

\begin{tabular}{|c|c|c|c|c|}
\hline \multirow{2}{*}{$\begin{array}{l}\text { Bead Type } \\
\text { Displacement }\end{array}$} & \multicolumn{2}{|c|}{ Plastic } & \multicolumn{2}{|c|}{ Glass } \\
\hline & $1^{\text {st }}$ & $2^{\text {nd }}$ & $1^{\text {st }}$ & $2^{\text {nd }}$ \\
\hline $\begin{array}{l}\text { Valid contact points } \\
\text { (number, } \% \text { of solid interface) }\end{array}$ & $1.22 \times 10^{6}, 5.18 \%$ & $1.42 \times 10^{5}, 0.60 \%$ & $3.22 \times 10^{5}, 1.50 \%$ & $2.78 \times 10^{5}, 1.30 \%$ \\
\hline $\begin{array}{l}\text { Invalid contact points } \\
\text { (number, \% of solid interface) }\end{array}$ & $1.72 \times 10^{6}, 7.31 \%$ & $1.42 \times 10^{5}, 0.60 \%$ & $5.84 \times 10^{5}, 2.73 \%$ & $1.16 \times 10^{5}, 0.54 \%$ \\
\hline $75^{\text {th }}$ percentile & $173.2^{\circ}$ & $170.3^{\circ}$ & $97.1^{\circ}$ & $95.5^{\circ}$ \\
\hline $50^{\text {th }}$ percentile & $165.1^{\circ}$ & $158.6^{\circ}$ & $54.8^{\circ}$ & $66.0^{\circ}$ \\
\hline $25^{\text {th }}$ percentile & $141.5^{\circ}$ & $139.1^{\circ}$ & $29.2^{\circ}$ & $42.4^{\circ}$ \\
\hline
\end{tabular}

Table 2 - Contact angle statistics for the mixed-wetting experiment

\begin{tabular}{lcccc}
\hline $\begin{array}{l}\text { Bead Type } \\
\text { Displacement }\end{array}$ & $1^{\text {st }}$ & $2^{\text {nd }}$ & $1^{\text {st }}$ & Glass \\
\hline $\begin{array}{l}\text { Valid contact points } \\
\text { (number, } \% \text { of solid interface) }\end{array}$ & $1.95 \times 10^{3}, 0.02 \%$ & $1.87 \times 10^{5}, 2.22 \%$ & $2.75 \times 10^{5}, 2.06 \%$ & $2.97 \times 10^{5}, 2.22 \%$ \\
Invalid contact points & $1.30 \times 10^{4}, 0.15 \%$ & $3.66 \times 10^{4}, 0.44 \%$ & $1.18 \times 10^{6}, 8.83 \%$ & $5.64 \times 10^{4}, 0.42 \%$ \\
$\quad$ (number, $\%$ of solid interface) & $169.5^{\circ}$ & $114.0^{\circ}$ & $88.3^{\circ}$ & $116.0^{\circ}$ \\
$75^{\text {th }}$ percentile & $159.1^{\circ}$ & $82.1^{\circ}$ & $50.1^{\circ}$ & $81.4^{\circ}$ \\
$50^{\text {th }}$ percentile & $136.5^{\circ}$ & $50.9^{\circ}$ & $29.2^{\circ}$ & $47.9^{\circ}$ \\
$25^{\text {th }}$ percentile & & & \\
\hline
\end{tabular}


For each bead material, the measured contact angle distribution can be compared to the laboratory based sensile drop measure. It is important to keep in mind that the sensile drop techniques measure contact angles with respect to surrounding air while the contact angles measured within the two phase experiments are measured with respect to a surrounding fluid. Differences in interfacial tension between the brine/air interface used in the lab and the brine/kerosene interface used in the experiment lead to systematic differences in the measured contact angles. However, the laboratory based measurements provide a baseline, and the contact angles measured within the two phase experiments are expected to follow similar trends. Based on the sensitivity analysis above, the following input parameters were used to calculate contact angles in the experimental data. The search radius was set to $10 \%$ of the average bead diameter. This is equal to 3 voxels (as shown in Figure 2). Face connectivity was used to define the contact and interface voxels. Within that search volume, a minimum of 5 points is required to fit a plane to the interface. If there are less than 5 points within the search volume, then it is assumed that there is an insufficient number of points to represent the interface and the contact angle is not calculated at that point. For most interfaces, more than 5 points are available to fit a plane. With the experimental data used in this study, 15 points were available, on average. These input parameters resulted in a consistent set of contact angle calculations across the different experimental data.

The median contact angles calculated in the uniform plastic bead pack after the $1^{\text {st }}$ and $2^{\text {nd }}$ displacements are $165.1^{\circ}$ and $158.6^{\circ}$, respectively (solid and dotted red lines in Figure 6a). For reference, the contact angle determined in the laboratory test is $100.9^{\circ}$. Despite this difference, which could be attributed to differences in the surrounding fluid or numerical error, the in situ measurement and lab test suggest that the plastic material is non-wetting. As expected, the contact angle distributions are also very similar after both displacements. This illustrates that the algorithm is able to compute similar contact angles in datasets with very different fluid geometries. The median contact angles calculated in the uniform glass bead pack after $1^{\text {st }}$ and $2^{\text {nd }}$ displacements are $54.8^{\circ}$ and $66.0^{\circ}$, respectively (solid and dotted blue lines in Figure 6a). These contact angles are also higher than the dynamic contact angle measurement of $47.7^{\circ}$. As seen with the plastic contact angle distributions, the contact angle distribution after both displacement cycles are very similar.

For the mixed glass/plastic bead pack, the contact angle can be associated with either glass or plastic. Median contact angles on the plastic beads are $159.1^{\circ}$ and $82.1^{\circ}$ after the $1^{\text {st }}$ and $2^{\text {nd }}$ displacements, respectively (solid and dotted red lines in Figure $6 \mathrm{~b}$ ). In contrast to the results seen in the uniform plastic core, the median contact angle associated with plastic beads in the mixed-wet core dropped dramatically after the $2^{\text {nd }}$ displacement and became intermediately wet. Contact angles associated with glass beads also show a shift towards intermediate wet after the $2^{\text {nd }}$ displacement. The contact angles on the glass beads had median values of $50.1^{\circ}$ and $81.4^{\circ}$ after $1^{\text {st }}$ and $2^{\text {nd }}$ displacements, respectively (solid and dotted blue lines in Figure $6 \mathrm{~b}$ ). After the $2^{\text {nd }}$ displacement in the mixed-wet system, contact angle distributions for both plastic and glass beads became almost identical with only $0.7^{\circ}$ separating the median values. This effect is thought to be caused by rock-fluid interactions due to connected residual fluid blobs in contact with both water-wet and oil-wet beads.

In the mixed glass/plastic bead pack, the residual fluid (brine) is mostly in contact with glass beads and fills small pore spaces after the $1^{\text {st }}$ displacement (see Figure 1 ). The small number of contact points associated with plastic beads after the $1^{\text {st }}$ displacement ( $1 \%$ of contact points) illustrates that the brine is rarely in contact with plastic beads at that point in the experiment. After the $1^{\text {st }}$ displacement, the glass and plastic beads have distinct contact angle distributions and those distributions are very similar to the glass and plastic contact angle distributions from the uniform wettability cores. After the $2^{\text {nd }}$ displacement, the residual fluid (kerosene) is in contact with glass and plastic beads and fills a range of pore configurations (see Figure 1). At this point in the experiment, the larger residual blobs are in contact with both glass and plastic beads. Contact points are more evenly divided between plastic and 
glass beads ( $61 \%$ and $39 \%$ respectively) and the contact angle distributions are very similar. This may explain why the residual fluid after the $2^{\text {nd }}$ displacement is just as likely to reside on glass or plastic beads.

While most contact angle measurements align with common understanding for wetting characteristics of glass and plastic beads, the distributions in Figure 6 show that there are a small number of contact angles greater than 90 degrees on glass beads and a small number of contact angles less than 90 degrees on plastic beads. Discrepancies between common understanding and the results could be attributed to complex porous media. While some of these values could be valid, some might suffer other sources of error, include error introduced by the segmentation algorithm and resolution. Furthermore, the fitting parameters used to compute the contact angle might not be optimal for every geometry encountered in the system.

It is clear that contact angles vary within a core sample and are not accurately represented by a single contact angle as determined by flat surface tests. Contact angle hysteresis, equilibrium time, heterogeneous surface properties, rock-fluid interactions, and $x$-ray exposure can all play a role in determining the distribution of contact angles within the system [11, 20-22]. Each bead pack underwent a series of displacement cycles which can change the advancing and receding contact angles. During each stage, the core was exposed to the infiltrating fluids at one end of the core. This means that each bead was exposed to the advancing fluid for different amounts of time. Since contact angles on a surface can be time dependent, this effect could cause a difference in contact angles within the bead pack. While all the beads are relatively homogeneous, slight differences in mineralogy and surface roughness could affect contact angles. Complex rock-fluid interactions including chemical reaction can also alter the wettability of surfaces. Segmentation methods also impact the contact angle calculation. It is well known that phase boundaries are very sensitive to segmentation criteria. A review of segmentation methods is included in [16]. As shown with the spherical test case, data resolution can also impact the contact angle calculation. While the test case illustrates that the experimental resolution is adequate to replicate a wide range of contact angles, the $90 \%$ confidence interval is just over $9^{\circ}$ when the bead is spherical and the interface is flat. This error is expected to propagate throughout the experimental results. Even though the experimental data includes many complexities, the algorithm is able to distinguish wettability characteristics in very different bead packs.

\section{Conclusions}

This paper presents an automated algorithm to measure in situ 3D contact angles. The algorithm was verified using a spherical test case over a range of resolutions and further demonstrated using micro-CT images of pore-scale multiphase experiments. The contact angle algorithm can be applied to any 3D data set with distinct solid and fluid phases. However, the accuracy of the algorithm would have to be thoroughly tested when applied to datasets with different resolution, segmentation, and different pore geometries. The current study uses spherical beads; systems with different packing structures will result in different accuracy and new test cases would have to be developed. User defined parameters, including the search radius, the minimum number of points needed to fit a plane, and type of connectivity used can be tuned to the desired system. Future research will combine contact angle measurements with pore characterization and different segmentation methods.

As technology advances, so will the quality of micro-CT scans. Higher resolution scans would allow for more accurate contact angle measurements in both bead pack and natural samples. The test case demonstrates that doubling the resolution greatly increases the accuracy of the 3D calculation. The resolution of CT scans could also be artificially increased using mathematical algorithms. These algorithms must be able to accurately retain core features without adding artifacts. Also, converting regularly gridded data to an irregular grid may increase the resolution of the interfaces. Using an irregular grid could also aid in memory usage as point density is a function of interface curvature [26]. 
Measuring in situ contact angles in different types of core samples would allow researchers to define more realistic contact angle distributions, which include natural variability and uncertainty, for reservoir characterization. These distributions could then be used to more accurately predict multiphase fluid flow. Mixed-wet systems that have undergone fluid displacement cycles are likely to have a wide range of contact angles and should not be considered to be uniformly wetting or nonwetting.

\section{Acknowledgements}

The authors would like to acknowledge funding support from the U.S. Department of Energy, the Office of Science, Basic Energy Sciences program under Award Number DE-SC0006883. We also thank Victor Torrealba (The Pennsylvania State University) and Jesse Roach (Tetra Tech) for their technical comments. Sandia National Laboratories is a multi-program laboratory managed and operated by Sandia Corporation, a wholly owned subsidiary of Lockheed Martin Corporation, for the U.S. Department of Energy's National Nuclear Security Administration under contract DE-AC04 94AL85000.

\section{References}

[1] Pope G, M Braviere. Reduction of capillary forces by surfactants. Basic Concepts in Enhanced Oil Recovery Processes. 33 (1991).

[2] NETL. Mobility and conformance control for carbon dioxide enhanced oil recovery $\left(\mathrm{CO}_{2}-\mathrm{EOR}\right)$ via thickeners, foams, and gels - A detailed literature review of 40 years of research. NETL-DOE2012.

[3] DePaolo DJ, DR Cole. Geochemistry of Geologic Carbon Sequestration: An Overview. Reviews in Minerology \& Geochemistry. 77 (2013) 1-14.

[4] Parmigiani A, C Huber, O Bachmann, B Chopard. Pore-scale mass and reactant transport in multiphase porous media flows. Journal of Fluid Mechanics. 686 (2011).

[5] Iglauer S, A Paluszny, $\mathrm{CH}$ Pentland, $\mathrm{MJ}$ Blunt. Residual $\mathrm{CO}_{2}$ imaged with X-ray micro-tomography. Geophysical Research Letters. 38 (2011) L21403, doi: 10.1029/2011GL049680.

[6] Tokunaga TK, J Wan. Capillary Pressure and Mineral Wettability Influences on Reservoir $\mathrm{CO}_{2}$ Capacity. Reviews in Minerology \& Geochemistry. 77 (2013) 481*503.

[7] Gray WG, CT Miller. TCAT analysis of capillary pressure in non-equilibrium, two-fluid-phase, porous medium systems. Advances in Water Resources. 34 (2011) 770-8, doi: http://dx.doi.org/10.1016/j.advwatres.2011.04.001.

[8] Zisman WA. Relation of the Equilibrium Contact Angle to Liquid and Solid Constitution. Contact Angle, Wettability, and Adhesion. AMERICAN CHEMICAL SOCIETY1964. pp. 1-51.

[9] Surface Science Techniques. Springer Berlin Heidelberg, 2013.

[10] Wenzel RN. RESISTANCE OF SOLID SURFACES TO WETTING BY WATER. Industrial \& Engineering Chemistry. 28 (1936) 988-94, doi: 10.1021/ie50320a024.

[11] Wan J, Y Kim, TK Tokunaga. Contact angle measurement ambiguity in supercritical $\mathrm{CO}_{2}$-water-mineral systems: Mica as an example. International Journal of Greenhouse Gas Control. 31 (2014) 128-37, doi: http://dx.doi.org/10.1016/j.ijggc.2014.09.029.

[12] Morrow NR. The Effects of Surface Roughness On Contact: Angle With Special Reference to Petroleum Recovery. (1975), doi: 10.2118/75-04-04.

[13] Liu Y, DD Nolte, LJ Pyrak-Nolte. Hysteresis and interfacial energies in smooth-walled microfluidic channels. Water Resources Research. 47 (2011) W01504, doi: 10.1029/2010WR009541.

[14] Broseta D, N Tonnet, V Shah. Are rocks still water-wet in the presence of dense $\mathrm{CO}_{2}$ or $\mathrm{H}_{2} \mathrm{~S}$ ? Geofluids. 12 (2012) 280-94, doi: 10.1111/j.1468-8123.2012.00369.x.

[15] Gao L, TJ McCarthy. Contact Angle Hysteresis Explained. Langmuir. 22 (2006) 6234-7, doi: 10.1021/la060254j.

[16] Wildenschild D, AP Sheppard. X-ray imaging and analysis techniques for quantifying pore-scale structure and processes in subsurface porous medium systems. Advances in Water Resources. 51 (2013) 217-46, doi: http://dx.doi.org/10.1016/j.advwatres.2012.07.018.

[17] Andrew M, B Bijeljic, MJ Blunt. Pore-scale imaging of geological carbon dioxide storage under in situ conditions. Geophysical Research Letters. 40 (2013) 3915-8, doi: 10.1002/grl.50771. 
[18] Celauro JG, VA Torrealba, ZT Karpyn, KA Klise, SA McKenna. Pore-scale multiphase flow experiments in bead packs of variable wettability. Geofluids. 14 (2014) 95-108.

[19] Torrealba VA, ZT Karpyn, H Yoon, KA Klise, D Crandall. Pore-scale imaging of geological carbon dioxide storage under in situ conditions. .

[20] Armstrong RT, ML Porter, D Wildenschild. Linking pore-scale interfacial curvature to column-scale capilary pressure. Advances in Water Resources. 46 (2012) 55-62.

[21] Andrew M, B Bijeljic, MJ Blunt. Pore-scale contact angle measurements at reservoir conditions using X-ray microtomography. Advances in Water Resources. (2014) 24-31.

[22] Brown K, S Schluter, A Sheppard, D Wildenschild. On the challenges of measuring interfacial characteristics of three-phase fluid flow with x-ray microtomography. Journal of Microscopy. 253 (2014) 171-82, doi: $10.1111 /$ jmi.12106.

[23] Landry CJ, ZT Karpyn, M Piri. Pore-scale analysis of trapped immiscible fluid structures and fluid interfacial areas in oil-wet and water-wet bead packs. Geofluids. 11 (2011) 209-27, doi: 10.1111/j.1468-8123.2011.00333.x.

[24] Hsieh J. Computed Tomography, Second Edition: Principles, Design, Artifacts, and Recent Advances, 2009.

[25] Cho-Chun C, P Guan-Ju, H Wen-Liang. Subband Weighting With Pixel Connectivity for 3-D Wavelet Coding. Image Processing, IEEE Transactions on. 18 (2009) 52-62, doi: 10.1109/TIP.2008.2007067.

[26] Thacker WC. A brief review of techniques for generating irregular computational grids. International Journal for Numerical Methods in Engineering. 15 (1980) 1335-41, doi: 10.1002/nme.1620150906. 